\title{
INTER Y TRANSDISCIPLINARIEDAD: UNA REFLEXIÓN EN LA EDUCACIÓN
}

Hugo González Aguilar

\section{Resumen}

En el presente artículo se analiza la inter y transdisciplinariedad desde un enfoque integrador, porque los problemas de la ciencia no se deben desarrollar de manera individual, aislada, sino en integración con las demás. Se analiza las concepciones y las propuestas para desarrollar la transdisciplinariedad.

Palabras clave: Interdisciplinariedad, transdisciplinariedad, investigación, problema, educación.

\section{Abstract}

This article discusses the inter and transdisciplinarity from an integrative approach, because the problems of science should not be developed for individual, isolated way, but integration with the other. Conceptions and proposals to develop transdisciplinary analyzes.

Keywords: Interdisciplinary, transdisciplinary, research, problem, education. 


\section{INTRODUCCIÓN}

La educación, desde los albores de la humanidad, ha ido transformándose, pero a la vez ha ido siendo estudiada desde diversas perspectivas y enfoques. Pero lo más importante: ha ido consolidando un tipo de hombre y un tipo de sociedad.

Así por ejemplo, en la comunidad primitiva la educación se realizaba de manera asistemática, natural, en la que los mayores o los padres enseñaban a sus hijos desde la práctica o desde el oficio que realizaban, por eso los hijos perennizaban las actividades de sus padres.

En la sociedad esclavista, es un tipo de educación segmentada, excluyente, en la que solo tenían la posibilidad de educarse los esclavistas, mas no los esclavos, quienes en realidad no tenían libertad para nada pues incluso no eran libres de sus propias vidas.

En la época medieval, el sistema casi no se altera, en relación al modelo anterior (el esclavista). Luego, en el capitalismo (con sus distintos modelos hasta llegar al neoliberalismo), en la escuela ha impuesto también cierta exclusión, bajo su modelo de inclusión.

Terminológicamente, suena interesante la inclusión educativa, sobre todo en nuestro país, pero vemos que muchos niños en la edad escolar no van a la escuela porque tienen que trabajar para sobrevivir; por otro lado, solo se está consolidando la educación de calidad limitadamente en algunos colegios, sobre todo los privados, en desmedro de los públicos, que están siendo olvidados por el Estado de una manera sistemática. Esto significa que si en el Perú se demanda una educación de calidad, esto representaría un costo elevado y, por tanto, no todos tendrían acceso a ella.

En este sentido, se requiere un estudio de la educación peruana desde la transdisciplinariedad, es decir, ser estudiada desde las diversas ciencias: económicas, sicológicas, pedagógicas, didácticas, sociológicas, políticas (la politicología, no la política partidaria, que ha hecho mucho daño al país), etc.

En este sentido, el presente artículo consolida la interdisciplinariedad y la transdisciplinariedad en el estudio del sistema educativo peruano.

No obstante, también se debe realizar un análisis del tránsito de la inter a la transdisciplinariedad. En este sentido, las ciencias o disciplinas han pasado desde un enfoque monodisciplinar, interdisciplinar, a uno transdisciplinar, lo cual significa que van desde los estudios individualistas (aislados, segmentados) a los estudios integrativos, pero sobre todo a los estudios que enfocan de manera integral y total la realidad compleja, que es el fin de los estudios transdisciplinarios.

\section{DESARROLLO}

La inter y transdisciplinariedad han surgido como un cambio en los estudios científicos, en lso cuales integran areas o disciplinas para estudiar de manera conjunta con más profundidad y amplitud la realidad,. 
A continuación, presentamos un cuadro donde se refleja las distintas concepciones o definiciones de diversos autores:

\section{Cuadro 1. Definiciones de inter y transdisciplinariedad}

\begin{tabular}{|c|c|c|c|}
\hline Disciplinas & $\begin{array}{l}\text { Autor, institu- } \\
\text { ción y año }\end{array}$ & Definición & Aplicación \\
\hline \multirow{7}{*}{$\begin{array}{l}\text { Interdisci- } \\
\text { plinariedad }\end{array}$} & $\begin{array}{l}\text { Mario Tamayo } \\
\text { y Tamayo, } \\
2004\end{array}$ & $\begin{array}{l}\text { Conjunto de disciplinas conectadas } \\
\text { entre sí. “...incorpora los resultados } \\
\text { de las diversas disciplinas (...) para } \\
\text { integrarlas"(Tamayo, } 2004 \text {, p. 67). }\end{array}$ & $\begin{array}{l}\text { El autor lo ha } \\
\text { desarrollado } \\
\text { en el marco } \\
\text { teórico de la } \\
\text { ciencia. }\end{array}$ \\
\hline & CIRET, 1994 & $\begin{array}{l}\text { Trata de la trasferencia de métodos } \\
\text { entre disciplinas... sus objetivos aún } \\
\text { permanecen en el seno de la trama de } \\
\text { las investigaciones disciplinares. }\end{array}$ & $\begin{array}{l}\text { Relación en- } \\
\text { tre disciplinas } \\
\text { (ciencia) }\end{array}$ \\
\hline & $\begin{array}{l}\text { Otros (segun- } \\
\text { da lectura) }\end{array}$ & $\begin{array}{l}\text { Establece transferencia de conoci- } \\
\text { mientos, habilidades, propósitos, ins- } \\
\text { trumentos y tareas. }\end{array}$ & $\begin{array}{l}\text { A la acción o lo } \\
\text { pragmático. }\end{array}$ \\
\hline & Martinez & $\begin{array}{l}\text { La coordinación, la comunicación, el } \\
\text { diálogo y el intercambio son esencia- } \\
\text { les, para traducir los términos pro- } \\
\text { pios, aclarar los lenguajes ambiguos, } \\
\text { seguir, aunque sea parcialmente, } \\
\text { procedimientos metodológicos simila- } \\
\text { res, y, en general, tratar de compartir } \\
\text { algunos de los presupuestos, puntos } \\
\text { de vista y lenguajes de los otros }\end{array}$ & $\begin{array}{l}\text { En las ciencias } \\
\text { sociales }\end{array}$ \\
\hline & Zavala, 2010 & $\begin{array}{l}\text { Interacción que produce subdiscipli- } \\
\text { nas } \\
\text { Interacción de dos o tres disciplinas } \\
\text { para estudiar un objeto específico }\end{array}$ & $\begin{array}{l}\text { In te r a c ción } \\
\text { entre discipli- } \\
\text { nas }\end{array}$ \\
\hline & $\begin{array}{l}\text { Max - Neef, } \\
2004\end{array}$ & $\begin{array}{l}\text { La interdisciplinaridad se organiza a } \\
\text { dos niveles jerárquicos }\end{array}$ & $\begin{array}{l}\text { Niveles de } \\
\text { aproximación } \\
\text { a la realidad } \\
\end{array}$ \\
\hline & $\begin{array}{l}\text { Nicolescu, } \\
1996\end{array}$ & $\begin{array}{l}\text { La interdisciplinariedad se refiere a la } \\
\text { transferencia de los métodos de una } \\
\text { disciplina a otra. }\end{array}$ & $\begin{array}{l}\text { Transferencia } \\
\text { de métodos a } \\
\text { otras discipli- } \\
\text { nas }\end{array}$ \\
\hline
\end{tabular}




\begin{tabular}{|c|c|c|c|}
\hline & $\begin{array}{l}\text { Mario Tamayo } \\
\text { y Tamayo, } \\
1999 .\end{array}$ & $\begin{array}{l}\text { Es un tipo de disciplinariedad. Conjunto } \\
\text { de disciplinas conexas entre sí (Tamayo, } \\
\text { 1999). }\end{array}$ & $\begin{array}{l}\text { Integración de } \\
\text { ciencias }\end{array}$ \\
\hline & $\begin{array}{l}\text { Mittlstrass, } \\
2003 \\
\end{array}$ & $\begin{array}{l}\text { Formas de investigación integrado- } \\
\text { ras. }\end{array}$ & En ciencia \\
\hline & $\begin{array}{l}\text { Nicolescu, } \\
2002\end{array}$ & $\begin{array}{l}\text { Unidad del conocimiento más allá de } \\
\text { las disciplinas. } \\
\text { Propone tres postulados metodoló- } \\
\text { gicos: la existencia de niveles de la } \\
\text { realidad, la lógica de los intermedios } \\
\text { incluidos y la complejidad. }\end{array}$ & En ciencia \\
\hline & $\begin{array}{l}\text { Hirsch Hadom } \\
\text { et al, } 2008 \\
\end{array}$ & $\begin{array}{l}\text { Integración conocimiento y práctica } \\
\text { promoviendo el bien común }\end{array}$ & $\begin{array}{l}\text { El mundo real } \\
\text { complejo }\end{array}$ \\
\hline & Aronson, 2003 & $\begin{array}{l}\text { Opera en el marco de estructuras di- } \\
\text { námicas y agrupamientos heterogé- } \\
\text { neos y transitorios. }\end{array}$ & En ciencia \\
\hline & CIRET, 1994 & $\begin{array}{l}\text { Su objetivo es la comprensión del } \\
\text { mundo actual. Uno de sus impera- } \\
\text { tivos es la unidad del conocimiento } \\
\text { global }\end{array}$ & En ciencia \\
\hline & $\begin{array}{l}\text { Planetary Col- } \\
\text { legium }\end{array}$ & $\begin{array}{l}\text { "El desarrollo del discurso transdis- } \\
\text { ciplinar en la convergencia de la in- } \\
\text { vestigación en el arte, la ciencia, la } \\
\text { tecnología y la conciencia" }\end{array}$ & $\begin{array}{l}\text { Aplicación en } \\
\text { arte y humani- } \\
\text { dades }\end{array}$ \\
\hline & Inglott, 1999 & Abordaje transdisciplinario en salud & $\begin{array}{l}\text { En problemas } \\
\text { de salud }\end{array}$ \\
\hline & Pedrosa & $\begin{array}{l}\text { Sugiere la colaboración entre las dis- } \\
\text { ciplinas, la interdependencia e inte- } \\
\text { gración. Se pretende la unidad de las } \\
\text { fuerzas y la apertura de las mentes. }\end{array}$ & $\begin{array}{l}\text { En todas las } \\
\text { ciencias }\end{array}$ \\
\hline & $\begin{array}{l}\text { D’Angelo, } \\
2002\end{array}$ & $\begin{array}{l}\text { Amplía el camino al diálogo y a las } \\
\text { múltiples relaciones entre sectores } \\
\text { del saber y del quehacer humanos } \\
\text { separados o distanciados a lo largo } \\
\text { de la vida }\end{array}$ & $\begin{array}{l}\text { En la teoría y } \\
\text { en la práctica, } \\
\text { relaciona el } \\
\text { ser y el hacer }\end{array}$ \\
\hline & Egilda, 2002 & $\begin{array}{l}\text { Comprender e interiorizar la exigen- } \\
\text { cia de organizar los conocimientos } \\
\text { con una visión cargada de relaciones, } \\
\text { ruptura de fronteras e integración a } \\
\text { partir de lo que todavía son discipli- } \\
\text { nas separadas. }\end{array}$ & $\begin{array}{l}\text { Su aplicación } \\
\text { a la educación } \\
\text { universitaria }\end{array}$ \\
\hline
\end{tabular}




\begin{tabular}{|c|c|c|c|}
\hline \multirow{6}{*}{$\begin{array}{l}\text { Transdisci- } \\
\text { plinariedad }\end{array}$} & Morin, 1994 & $\begin{array}{l}\text { La transdisciplinariedad es comple- } \\
\text { mentaria al enfoque disciplinario. } \\
\text { Busca la apertura de todas las dis- } \\
\text { ciplinas. Sus características princi- } \\
\text { pales son el rigor, la apertura y tole- } \\
\text { rancia. Es un diálogo no solo con las } \\
\text { ciencias humanas sino con el arte, la } \\
\text { literatura, la experiencia interior. } \\
\text { Se concibe como una visión del mun- } \\
\text { do que busca ubicar al hombre y a } \\
\text { la humanidad en el centro de nuestra } \\
\text { reflexión, y desarrollar una concepci- } \\
\text { ón integradora del conocimiento glo- } \\
\text { bal (década del 70). }\end{array}$ & $\begin{array}{l}\text { Integración } \\
\text { entre las cien- } \\
\text { cias }\end{array}$ \\
\hline & Martínez & $\begin{array}{l}\text { Va más allá de ellas (ciencias), y les } \\
\text { añade el hecho de que está constitui- } \\
\text { da por una completa integración teo- } \\
\text { rética y práctica. } \\
\text { Una "ciencia" transdisciplinaria y } \\
\text { transcendente se vuelve necesaria } \\
\text { para entender los amplios y comple- } \\
\text { jos sistemas del mundo actual }\end{array}$ & $\begin{array}{l}\text { Relación teoría } \\
\text { y práctica para } \\
\text { comprender la } \\
\text { realidad actual } \\
\text { que es com- } \\
\text { pleja }\end{array}$ \\
\hline & $\begin{array}{l}\text { Congreso de } \\
\text { Lucarno, Sui- } \\
\text { za, } 1997\end{array}$ & $\begin{array}{l}\text { La actitud transdisciplinar implica } \\
\text { la puesta en práctica de una nueva } \\
\text { visión transcultural, transnacional, } \\
\text { transpolítica y transreligiosa. }\end{array}$ & $\begin{array}{l}\text { Integración de } \\
\text { la ciencia con } \\
\text { otras áreas del } \\
\text { saber o del ha- } \\
\text { cer humano }\end{array}$ \\
\hline & Zavala, 2010 & $\begin{array}{l}\text { Independiente de la lógica disciplina- } \\
\text { ria. Preguntas que rebasan la especi- } \\
\text { ficidad de cada disciplina particular }\end{array}$ & $\begin{array}{l}\text { Van más allá de } \\
\text { la individualidad } \\
\text { de las ciencias }\end{array}$ \\
\hline & $\begin{array}{l}\text { Otros (docu- } \\
\text { mento siete) }\end{array}$ & $\begin{array}{l}\text { La transdisciplinariedad basa sus re- } \\
\text { flexiones en el "descubrimiento" de la } \\
\text { complejidad como punto de partida } \\
\text { para comprender la realidad social } \\
\text { contemporánea. }\end{array}$ & $\begin{array}{l}\text { Comprender } \\
\text { la realidad so- } \\
\text { cial contempo- } \\
\text { ránea que es } \\
\text { cambiante y } \\
\text { compleja }\end{array}$ \\
\hline & $\begin{array}{c}\text { Max - Neef, } \\
2004\end{array}$ & $\begin{array}{l}\text { La transdisciplinaridad se da cuando } \\
\text { existe una coordinación entre todos } \\
\text { los niveles: los que existe, lo que se } \\
\text { hace, lo que queremos hacer, lo que } \\
\text { debemos hacer. }\end{array}$ & $\begin{array}{l}\text { Integración de } \\
\text { las disciplinas } \\
\text { según sus fi- } \\
\text { nes }\end{array}$ \\
\hline
\end{tabular}




\begin{tabular}{|c|c|l|l|}
\hline & $\begin{array}{c}\text { Enríquez, } \\
2011\end{array}$ & $\begin{array}{l}\text { Los diálogos culturales y la colabora- } \\
\text { ción de las ciencias. }\end{array}$ & $\begin{array}{l}\text { Aplicación de } \\
\text { Derecho Com- } \\
\text { parado }\end{array}$ \\
\cline { 2 - 4 } & $\begin{array}{l}\text { Transdisci- } \\
\text { plinariedad }\end{array}$ & $\begin{array}{l}\text { La transdisciplinariedad comprende, } \\
\text { como el prefijo “trans" lo indica, lo } \\
\text { que está, a la vez, entre las discipli- } \\
\text { nas, a través de las diferentes disci- } \\
\text { plinas y más allá de toda disciplina. }\end{array}$ & $\begin{array}{l}\text { En todas las } \\
\text { disciplinas }\end{array}$ \\
\hline $\begin{array}{l}\text { Su finalidad es la comprensión del } \\
\text { mundo presente, y uno de sus impe- } \\
\text { rativos es la unidad del conocimiento. }\end{array}$ & \\
\hline
\end{tabular}

Fuente: Elaborado por el autor

Del cuadro precedente, consideramos que la inter y la transdisciplinariedad, en realidad no son antagónicas sino complementarias. La transdisciplinariedad profundiza y amplía sus fines, pretende resolver problemas más complejos con la integración de todas las ciencias. Por eso es más abarcadora, más holística, totalizadora.

En cambio, la interdisciplinaria comparte métodos de una disciplina con otras. Por ejemplo, los métodos de la lógica pueden ser aplicados a las ciencias jurídicas, sociales; pero su interés no es integrar las ciencias para resolver un problema, sino solo compartir el método.

Otro aspecto de la interdisciplinariedad puede ser la teoría que pueden compartir las ciencias, por ejemplo: la teoría matemática puede ser compartida con la física y, de esto, surgir la Física Matemática; en este sentido, también la Educación puede basarse en las teorias filosóficas y, de esto, surge la Filosofia de la Educación.

Por tanto, la transdisciplina difiere porque integra varias disciplinas para resolver un problema de manera integral, total; en este sentido, por ejemplo, la educación vista desde este enfoque debe estudiarse o recibir los aportes de la pedagogía, la didáctica, la sociología, la antropología, la psicología, la historia, la economía, puesto que es un problema complejo que incluso debemos evaluarlo desde su contexto histórico-social y cultural. Por el contrario, la educación vista desde la perspectiva interdisciplinaria, puede adoptar estrategias y métodos de la psicología para realizar los procesos didácticos o de enseñanza.

En este sentido, la interdisciplinariedad se convierte en antesala, en preámbulo de la transdisciplinariedad; empieza a estudiar la realidad de una manera integrada, relacionando teorías, métodos, prácticas, pero especificamente no ciencias; en cambio, la transdisciplinariedad integra ciencias, sus propósitos, sus fines, para estudiar la realidad de manera global, integral y no segmentándola.

En suma, son semejantes porque de una $u$ otra manera comparten el 
concepto de integración; pero son diferentes porque la transdisciplinariedad integra áreas y aborda la complejidad de la realidad de manera integral, holistica.

Reflexionando en el ámbito educativo, que nos corresponde como educadores, debemos enfocarla en todas sus dimensiones: política, económica, cultural, social, formativa, científica, filosófica, etc.

Entonces, ¿cómo enfrentar el reto de educar adecuadamente al ser humano desde la transdisciplinariedad?

Esta pregunta nos lleva a formar a una persona de manera global, integral; esto significa formar sus emociones, sentimientos, a enfrentar las incertidumbres propuestas por Morin, temores o sus demonios internos, como diria Goethe (a través de la psicologia); formar su pensamiento a través de la lógica, la matemática; formar o practicar valores a través de la ética, que ha venido a menos en estos últimos años; a formar o educar en la toma de decisiones sociales, o el poder colectivo a través de la Politologia; formar su habilidad comunicativa a través del Lenguaje o la Lingüística; formar su valoración hacia los demás, para aprender a convivir y a tolerar otras culturas, costumbres, a valorar su propia cultura a través de las ciencias sociales (Antropología, Etnología, Historia); formar su comunicación con otras culturas a través del aprendizaje de otros idiomas; formar su capacidad de selección y búsqueda de información a través de la tecnología, pero con dominio de procesos metodológicos científicos.
Como consecuencia, estaremos formando un ser integral que responda a una sociedad concreta, específica, con el propósito de mejorar las condiciones de vida del ser humano y no vivir en la segmentación, en la exclusión. A pesar de que en estos años los gobiernos de turno (en el Perú) están difundiendo en los medios que estamos en la era de la inclusión, esto es más excluyente porque sigue priorizándose la privatización de la educación, porque las personas de menores recursos económicos no tienen acceso a una educación de calidad pues esta es costosa; a diferencia de los centros educativos estatales, que yacen en el olvido por una pésima administración.

Las investigaciones transdisciplinarias deben tener como fin unificar la educación privada y estatal, que ambas den una educación de calidad, en la que no haya segregaciones, marginaciones, con el fin de que todos, a través de la educación, mejoren sus condiciones de vida, o simplemente tengan una vida digna.

Como se puede apreciar, la investigación transdisciplinaria en el ámbito educativo peruano está por construirse y no solo dependerá de la integración de las ciencias, sino que se requerirá la integración de los diversos sectores: industriales, empresariales, políticos, científicos, organizaciones o colegios profesionales, los mismos medios de comunicación que han dejado de cumplir un rol importante que es el de educar, más bien deseducan (parafraseando a Illich). 
En suma, para mejorar nuestro sistema educativo, en todos sus niveles, se requiere una integración de las ciencias, pero sobre todo de los sectores o actores sociales, incluyendo a los excluidos o quienes pertenecen al cuarto mundo (a decir de nuestro amauta Peñaloza) que son las comunidades nativas que son o siguen siendo olvidadas por el Estado y que están todavía en una etapa primitiva.

En consecuencia, la transdisciplinariedad, para el caso de la educación peruana, no solo se trata de integración de ciencias, sino de actores sociales, grupos sociales que respondan a sus propias realidades, necesidades, aspiraciones y no solo de imposiciones que se han realizado a través del $\mathrm{Mi}$ nisterio de Educación que impone un solo currículo, en un solo idioma frente a realidades que hablan en otros idiomas; urge un diálogo intercultural, étnico.

Considero, desde mi punto de vista, que la transdisciplinariedad se ajusta a nuestra realidad diversa, heterogénea porque es abierta, integradora, totalizadora, holística; evalúa los problemas contextualizados y no creados artificialmente (como los programas) como en las investigaciones de laboratorio o las tecnológicas.

La transdisciplinariedad bien estudiada y aplicada, que surja del compromiso de nuestros científicos educadores o educadores científicos y de nuestros actores sociales de todas las regiones, debería orientarnos a solucionar nuestro problema educativo que cada día va sumiéndose en el olvido, acompañado de la baja calidad, de la pobreza de los sectores que menos tienen.

La transdisciplinariedad debe aplicarse y practicarse desde arriba (del poder político o quienes lo dirige) $\mathrm{y}$ desde abajo (los que eligen pero que no tienen pode de decidir, pero sí de opinar).

\section{CONCLUSIONES}

- La transdisciplinariedad se complementa con la interdisciplinariedad, pero estudia la realidad de manera más profunda, integral, global.

- La transdisciplinariedad surge integrando ciencias para que respondan a una realidad compleja, diversa, heterogénea.

- La transdisciplinariedad para el sistema educativo peruano no solo comprende la integración de ciencias, sino de sectores sociales para solucionar los problemas educativos urgentes, entre ellos la exclusión educativa, la segregación social, sobre todo de las comunidades.

- El trabajo de Lauro "Transdisciplinariedad", refiere los retos de esta disciplina desde su historia, su etimología, que responda a las nuevas realidades e incluso a la misma pedagogía, que debe responder a la integración humana, que responda óptimamente a la formación humana integral. 


\section{REFERENCIAS BIBLIOGRÁFICAS}

Diaz, G. (s.a). Edgar Morin y los principios de la Transdisciplinariedad.

Enríquez, D. (2011). Interculturalismo y transdisciplinariedad: coordenadas en el mapa del Derecho. UNAM: Biblioteca Jurídica Virtual del Instituto de Investigaciones Jurídicas, de www.juridicas.unam.mx

Martínez Miguélez, M. (s. a). Un enfoque para la complejidad del mundo actual.

Morín, E. (1994). Carta de la Transdisciplinariedad. Convento de Arrábida.

Morín, E. (2005). El paradigma perdido. Ensayo de Bioantropología. Séptima edición. Barcelona: Editorial Kairós.

Nicolescu, B. (1996). La transdisciplinariedad. Manifiesto. México: Multiversidad Mundo Real Edgar Morin, A.C.

Tamayo y Tamayo, M. (1999). La investigación. Santa Fe de Bogotá: ARFO EDITORES LTDA.

Tamayo y Tamayo, M. (2004). El proceso de la investigación científica. Incluye evaluación y administración de proyectos de investigación. Cuarta edición. México: LIMUSA. 